INPLASY

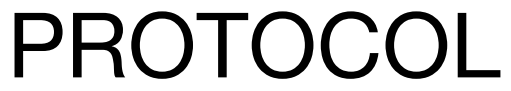

To cite: Zhan et al. Efficacy and safety of escitalopram and agomelatine in the treatment of major depressive disorder - A meta-analysis. Inplasy protocol 2021120074. doi:

10.37766/inplasy2021.12.0074

Received: 16 December 2021

Published: 16 December 2021

Corresponding author:

Wang Yunxia

cloudywang66@163.com

Author Affiliation:

Naval Medical University, China.

Support: National Natural Science Found.

Review Stage at time of this submission: Formal screening of search results against eligibility criteria.

\section{Efficacy and safety of escitalopram and agomelatine in the treatment of major depressive disorder - A meta-analysis}

Review question / Objective: The aim of this meta-analysis of randomized controlled trials is to evaluate the efficacy and safety of escitalopram and agomelatine in the major depressive disorder.

Condition being studied: Major depressive disorder (MDD), is one of the most common, costly, and disabling mental health conditions worldwide, with an estimated 246 million sufferers globally in 2020.At present, there is a great demand for effective antidepressant treatment in medicine.

Information sources: We will search, with no time restrictions, the following databases for relevant English language literature: PubMed, the Cochrane Central Register of Controlled Trials and Web of Science. The search string will be built as follows: (escitalopram) AND (agomelatine) AND (major depressed disorder).

INPLASY registration number: This protocol was registered with the International Platform of Registered Systematic Review and Meta-Analysis Protocols (INPLASY) on 16 December 2021 and was last updated on 16 December 2021 (registration number INPLASY2021120074).

Conflicts of interest:

None declared.

\section{INTRODUCTION}

Review question / Objective: The aim of this meta-analysis of randomized controlled trials is to evaluate the efficacy and safety of escitalopram and agomelatine in the major depressive disorder.

Condition being studied: Major depressive disorder (MDD), is one of the most common, costly, and disabling mental 
health conditions worldwide, with an estimated 246 million sufferers globally in 2020.At present, there is a great demand for effective antidepressant treatment in medicine.

\section{METHODS}

Participant or population: Patients with major depressive disorder (as defined by DSM-IV or HAM-D17) will be included. Patients with other critical illness (e.g. miocardial infarction, ARDS, etc.) will be excluded.

Intervention: Drug therapy was the main intervention (e.g. escitalopram).

\section{Comparator: Drug therapy (agomelatine).}

Study designs to be included: Randomized controlled trials (RCTs).

Eligibility criteria: (i)patients with major depressive disorder were defined by DSMIV or HAM-D17. (ii)use of the mean change from baseline in HAM-D17 total score as an efficacy measure. (iii)safety was reported after at least 8 weeks of treatment. (iv)double-blind, randomised, treatment period of at least 8 weeks.

Information sources: We will search, with no time restrictions, the following databases for relevant English language literature: PubMed, the Cochrane Central Register of Controlled Trials and Web of Science. The search string will be built as follows: (escitalopram) AND (agomelatine) AND (major depressed disorder).

Main outcome(s): the change of the HAMD17 total score from the baseline to the end.

Quality assessment / Risk of bias analysis: Two reviewers will independently assesses the quality of the selected studies according to the Cochrane Collaboration's tool for randomized controlled trials. Items will be evaluated in three categories: low risk of bias, unclear bias and high risk of bias. The following characteristics will be evaluated: Random sequence generation(selection bias) 、Allocation concealment (selection bias)、Blinding of participants and personnel (performance bias). Other biases Results from these questions will be graphed and assessed using Review Manager 5.3.

Strategy of data synthesis: Risk ratio for both fixed and randomized effects models will be used. Between-study heterogeneity will be assessed using the Cochrane $Q$ and $I^{2}$ statistics. According to the Cochran handbook, the $\mathrm{I}^{2}$ will be considered nonimportant $(<30 \%)$, moderate (30\%-60\%) and substantial $(>60 \%)$. Results will be assessed using forest plots. Publication bias will be assessed by a funnel plot for meta-analysis and quantified by the Egger's test. Statistical analyses were performed using STATA version 16.0 software(Stata Corp., College Station, Texas), and all statistical tests were twosided with the type I error set to 0.05 .

Subgroup analysis: We will consider subgroups such as duration of the drug therapy, sex rate and so on.

Sensitivity analysis: Parameters that were considered for this analysis were mean change in the HAM-D17 score, studies that employed only HAM-D17 scale and randomized effect model of analysis. Visual inspection of funnel plots was performed to investigate for the presence of publication bias.

Language: English.

Country(ies) involved: China.

Keywords: escitalopram; agomelatine; major depressive disorder.

Contributions of each author:

Author 1 - Zhan Yuji.

Email: nmuzhanyuji@163.com

Author 2 - Rui Mengxin.

Email: 455399717@qq.com

Author 3 - Zeng Wenfeng.

Author 4 - Wang Yunxia.

Email: cloudywang66@163.com 